\title{
From inflammation to cancer
}

\author{
A. Korniluk ${ }^{1} \cdot$ O. $\operatorname{Koper}^{1} \cdot$ H. Kemona ${ }^{1} \cdot$ V. Dymicka-Piekarska ${ }^{1}$
}

Received: 22 January 2016/Accepted: 23 April 2016/Published online: 7 May 2016

(c) The Author(s) 2016. This article is published with open access at Springerlink.com

\begin{abstract}
Background The participation of inflammation in the progression of cancer for many years have been the subject of research.

Methods In the 19th century, there was evidence that an acute inflammation may inhibit the development of cancer. However, chronic inflammation affects the progression of the disease.

Results Today, it is known that inflammation and cancer use similar mechanisms of development such as severe cell proliferation or angiogenesis. It has been shown that prolonged presence of inflammatory cells and factors in the tumor microenvironment can accelerate its growth and inhibit apoptosis of transformed cells.

Conclusion In this article we present a brief history of the discovery mechanisms and potential links between acute and chronic inflammation and cancer.
\end{abstract}

Keywords Acute inflammation - Chronic inflammation . Cancer - Adhesion molecules

\section{Introduction}

In 1863, Rudolf Virchow put forward a hypothesis that infiltrated immune cells reflect the place where cancer lesions appear in the inflamed tissue [1]. Not earlier than a century later, Dvorak showed that carcinogenesis and

V. Dymicka-Piekarska

piekarskav@yahoo.com

1 Department of Clinical Laboratory Diagnostics, Medical

University of Bialystok, Ul. Waszyngtona 15A,

15-276 Bialystok, Poland inflammatory conditions have common developmental pathways, such as proliferation, increased cell survival and migration, and enhanced angiogenesis which are controlled by growth factors, proinflammatory cytokines and proangiogenic factors. Moreover, the author observed that the cells involved in inflammation also infiltrated cancer tissue $[2,3]$ and defined cancer as a "wound that does not heal". Therefore, we believe that in order to fully elucidate the role of an inflammatory condition in carcinogenesis, first we should have a profound understanding of what inflammation really is.

\section{Short brief history}

The discovery and understanding of inflammatory mechanisms dates back to the ancient times of Egyptians and Greeks. The first description of inflammation appears in the works of Aulus Cornelius Celsus, who defined four basic inflammatory signs and symptoms: redness, swelling, warmth and pain. In 2nd century $\mathrm{AD}$, a Roman surgeon Galen defined the inflammatory state as a favorable early component of healing processes in injured tissues, leading to recovery. Some authors suggest that he introduced the fifth sign of inflammation, i.e., function loss. However, according to most scientists, the term functio laesa was introduced in 1871 by Virchow [4].

Although ancient scientists based their concepts mainly on observations and intuition and not on profound investigations, they initiated later experiments aimed to discover the mechanisms involved in the inflammatory process. The invention of a microscope was a breakthrough in their understanding, making it possible to observe the microcirculatory changes in inflamed tissues [5]. For instance, blood clotting "tendency" was discovered (Gaubius 1794) and 
angiogenesis was first described as the process of new vessel formation in healing tissue. In 1824, Dutrochet showed the ability of leukocytes to migrate within the blood and 15 years later Weyner described the process of leukocyte rolling on the endothelium. Molecular changes in the vascular walls were found to underlie the adhesion of leukocytes to the endothelium. In nineteenth century, Virchov propagated research into an inflammatory state [6]. Contrary to Galen, he described the inflammatory condition as a pathological process associated with the excessive inflow and proliferation of cells caused by the release of nutrients from damaged blood vessels. He was the first to observe the cellular nature of an inflammatory response. He also emphasized the involvement of leukocytes in inflammation, although he erroneously concluded that these cells were responsible for infection and tissue damage. A Virchov's student, Cohnheim (1867) suggested that the increased "porosity" of blood vessels is associated with fluid escape to the extravascular space. Using a microscope he was the first to describe the sequences that occur during an inflammatory condition; however, like his master, he wrongly defined the functions of leukocytes [7]. It was only a Russian scientist, Miecznikow, who refuted these theories and proved that it was the invasion of a foreign agent that caused the migration of host cells to the infected site, phagocytosis and damage of pathogens. In the subsequent years, the role of inflammatory cells (including phagocytes) was described, whereas research conducted by Rocha e Silva showed the involvement of "chemical mediators" in the development of an inflammatory condition, which supplemented the knowledge of an inflammatory response [4].

Currently, we know that irrespective of the triggering agent, tissue damage activates signals that initiate and support the immune response of the host, able to eliminate a damaging agent and to repair tissues [8]. Pathogen invasion activates inflammatory cells, macrophages and mast cells that generate and release cytokines (TNF- $\alpha$, IL$1 \beta$, IL-6) and proinflammatory chemokines (IL-8, MCP-1, MIP-1 $\alpha$ ), which together with chemoattractants (bacterial toxins) stimulate attraction of circulating leukocytes to the site of inflammation [9].

\section{Inflammatory condition step by step}

The initial stage of an inflammatory response involves leukocyte marginalization. It takes place in small-diameter vessels, in which inflammatory cells do not migrate to the center but are pushed towards the vascular walls, where the process of their endothelial rolling begins. TNF- $\alpha$, histamine and other mediators of inflammation stimulate translocation of P-selectin from Weibel-Palade corpuscles, which is later incorporated in the endothelial cell membrane. Selectin interacts with sialylated or fucosylated carbohydrate residues of the Lewis group antigens, which act as their ligands. PSGL-1 is best known among them [10]. However, any adequately modified glycoprotein can be a potential selectin receptor, like CD34, which having been transformed on the endothelial surface becomes L-selectin receptor on leukocytes [11]. Ligand binding to selectin is a dynamic process and the bonds being formed undergo rapid dissociation. Many times the binding of leukocytes to endothelium slows down their vascular flow from 1000 to $30 \mu \mathrm{m} / \mathrm{s}$. A few hours after activation, E-selectin appears on the endothelial surface [12]. P-selectin mediates binding at high shear stress, whereas E-selectin takes part in slow leukocyte rolling (low shear stress). This is associated with partial leukocyte integrin activation [13]. The rolling leukocytes may have a direct contact with endothelium, due to which they may be activated by inflammatory factors (chemokines) present on the endothelial surface.

Chemokines are produced by endothelial cells or interstitial inflammatory cells and then transported to the vascular lumen, where they bind to heparin sulfate glycosaminoglycans on the endothelial surface. Contact of these proteins with specific leukocyte receptors (a subgroup of receptors conjugated with $\mathrm{G}$ protein) causes the transmission of a signal that activates integrins present on the inflammatory cells [14].

Integrins belong to a family of heterodimeric adhesion receptors that remain in the resting inactive conformation. Most integrins are the extracellular matrix proteins. During activation changes take place in the arrangement of these proteins, which allows binding to a ligand [15]. Leukocytes have on their surface members of the $\beta 2$ family (CD18), whose main ligands, i.e., the immunoglobulin-like molecules, ICAM-1 and ICAM-2, are present on the endothelium [16]. The surface of lymphocytes and monocytes exhibits the $\beta 1$ family integrins (VLA-4, CD49d/CD29, $\alpha 4 \beta 1$ ), which bind to VCAM-1 molecules [17]. Following activation, integrins bind leukocytes tightly to the endothelial surface, causing their complete arrest. Next, leukocytes "crawl" to the contact point of endothelial cells where they prepare for extravasation. The process of "crawling" is mediated by proteins belonging to the leukocyte and endothelial integrins, i.e., Mac-1 (CD11b/ CD18) that bind ICAM-1. In the case of monocytes, the transmigration is mediated also by LFA-1 (CD11a/CD18) and ICAM-2 [18]. During diapedesis leukocytes assume the amoeboid shape as a result of cytoskeletal alterations and push their way between endothelial cells [19]. The stages of rolling, activation, adhesion and "crawling" are reversible and require heterophilic bonds. On the contrary, diapedesis is a no-return path, during which leukocytes interact due to homophilic bonds [20]. 
PECAM-1 is the first molecule reported to take part in transmigration (TEM). It shows a specific distribution on the cell surface. PECAM-1 on leukocytes has been found dispersed throughout the cell membrane, whereas on endothelial cells its highest level has been shown at the cell contact point. Apart from PECAM-1, such distribution is also characteristic of CD99 protein [21, 22]. Leukocytes' migration to the extravascular space is associated with the fact that they have to pass through the interstitial tissue. It has been suggested that neutrophils and monocytes migrate through the basement membrane to the sites with the lowest expression of collagen IV and laminin 10. This process is to delimit injuries caused by proteolytic enzymes used by leukocytes during their passage to the site of damage. The process occurs when the inflammatory cell CD11a binds to pericyte-ICAM-1 to allow leukocytes to reach the sites with the lowest density of the collagen network [23]. Having reached the site of damage, leukocytes eliminate the pathogen through the activation of aerobic and anaerobic killing. If the process runs properly, the elimination of the inflammatory factor suppresses the inflammation thanks to the action of anti-inflammatory mediators. For instance, the production of proinflammatory prostaglandins is decreased and the generation of anti-inflammatory lipoxins that attract monocytes, IL-10 or TGF, is increased [24].

Neutrophils which appear early at the site of damage constitute the first line of body defense. To a large extent, they are able to eliminate a pathogen on their own. Other cells include monocytes and lymphocytes initiating healing and generation of immunological memory [25].

When the inflammatory cells are not able to eliminate a pathogen, acute inflammatory condition may turn chronic. This chronic phase is characterized by hyper-intensive leukocyte infiltration of injured tissues, which results in the formation of leukocyte infiltrates. They were first described by Virchov when he examined tumor tissues. He observed that the "lymphoreticular infiltrate reflected the origin of cancer at sites of chronic inflammation" [1]. The elucidation of the mechanisms responsible for the development of inflammation initiated the research into mutual relationship to neoplastic disease [26].

\section{Acute inflammatory condition: not always bad}

The fact that inflammation can have a double role in the development of cancer was already observed in the 19th century (1868) by a German physician Burns. He described cases of severe streptococcal infection during which patients experienced regression of neoplastic disease. More than 20 years later, this fact was taken into consideration by Coley who created the so called "Coley's toxin", a suspension of gram-negative bacterial cells which he successfully administered to cancer patients. The pathogeninduced body stimulation results in the activation of the immune system, which not only fights the bacteria but also inhibits tumor growth. The role of TNF- $\alpha$ (tumor necrosis factor), whose release is stimulated, among others, by a bacterial toxin, LPS (lipopolysaccharide), has been proved [27]. Currently, induced acute inflammation is used in the therapy of squamous cancer of the bladder. Patients receive a special vaccine against tuberculosis containing an attenuated Mycobacterium bovis strain (Mycobacterium bovis bacillus Calmette-Guerin-BCG) directly to the urinary bladder [28]. Induced immune reaction is associated with cytokine secretion, including interleukin-1 (IL-1), IL-2, IL5, IL-6, IL-8, IL-10, IL-12, IL-15, IL-18, TNF- $\alpha$, GM-CSF and interferon (IFN) $-\gamma$ [28]. Studies are being conducted on the potential use of Salmonella typhimurium containing vaccines in oncological therapy. Acute inflammatory condition is considered a physiological process and a body's defensive response. Sometimes referred to as "therapeutic inflammation" can show anticancer effects. However, it should be remembered that pathogens try to avoid the defense systems of the host, which may lead to a chronic condition that often accompanies the neoplastic disease.

\section{Chronic inflammatory condition: always bad}

"Pathological inflammation", i.e., acute phase response of moderate intensity (chronic inflammation) is involved in neoplastic transformation and stimulation of cancer growth [29]. Already in 1828, Jean Nicholas Marjolin, a French surgeon, provided evidence for the involvement of chronic inflammation in the development of cancer. He observed the growth of squamous cancer around the open chronically inflamed wound. It is currently estimated that approximately $25 \%$ of cancers are associated with chronic inflammation caused by infection or physicochemical agents [30]. Persistent gastritis induced by Helicobacter pylori increases the risk of stomach cancer even by $75 \%$ [31], whereas types $\mathrm{B}$ and $\mathrm{C}$ hepatitis promote the formation of hepatocellular carcinoma [29]. Also noninfectious diseases may increase the risk of cancer. Cancers of the pancreas and prostate often follow chronic inflammation in these organs [32]. The closest relationship between chronic inflammation and cancer can be observed in chronic ulcerating colitis and Crohn's disease, which increase the risk of colorectal cancer even tenfold [33]. Moreover, inflammation-based tumors are characterized by the presence of cells and inflammatory mediators, thus indicating that inflammation and cancer can be combined by the extrinsic and intrinsic pathways. In the former, cancer growth is associated with an ongoing inflammatory state. 
The latter is stimulated by genetic changes leading to the activation of oncogenes and inactivation of suppressor genes. Cells with an altered phenotype produce inflammatory mediators thus leading to the activation of immune response and development of inflammation [34].

In both models, carcinogenesis is a two-stage process. Cancer development is initiated when the DNA sequence in somatic cells undergoes genetic mutations that can be present in healthy tissues and remain occult for many years until another stimulus pushes the cells into the "promotion" stage [30]. This process may occur when the transformed cells are exposed to chemical irritants, such as phorbol esters, substances released at the site of injury, partial resection of the organ, hormones or chronic irritation and inflammation. Functionally, many "promoters" may directly or indirectly induce cell proliferation, recruitment of inflammatory cells, may increase production of reactive oxygen species, leading to oxidative DNA damage and decreasing its repair [35].

In the process of initiation normal cells undergo mutation. Usually a single change in DNA is insufficient, but 4-5 mutations can direct cells onto the neoplastic transformation pathway. Activated leukocytes release reactive oxygen species (ROS) and reactive nitrogen intermediates (RNI), which can induce DNA damage and genome instability [8]. The major role of inflammation in mutagenesis can be exemplified by the development of colorectal adenocarcinoma induced by inflammation and the action of an irritant, dextran sodium sulfate (DSS) [35]. DSS itself shows a weak carcinogenic effect, although the inflammatory state highly augments the effect [35]. Moreover, the inflammatory cells producing cytokines may induce overexpression of cytidine deaminase (activation-induced cytidine deaminase-AID). AID causes genome instability and increases the probability of mutations in the course of abnormal binding of double stranded DNA, i.e., when most frequently mutations form in oncogenesis-critical genes, such as TP53, c-myc and Bcl-6. AID involvement has been demonstrated in the formation of lymphomas, and gastric and liver cancers [36]. Damage to DNA can also lead to the inflammatory process and in consequence to cancer formation. The examples include DEN-induced hepatocellular carcinoma induced by DEN (diethylnitrosamine), which causes DNA damage resulting in necrotic cell death and inflammatory response that promotes cancer progression [37]. Many oncoproteins (RAS, Myc, RET) can also activate the signaling pathways which enhance the production of inflammatory cytokines and chemokines (IL-6, IL-8, IL$1 \beta$, CCL2, CCL20) [34]. The initiation stage lasts until an additional factor begins the stage of promotion, and thus growth from single cells to a fully developed primary cancer. The initial growth of cancer depends on the increased cell proliferation and limited apoptosis, and these two processes are stimulated by mechanisms closely related to inflammation. In fact, most inflammatory mediators that affect the growth of cancer act during the stage of promotion. Moreover, inflammation-inducing factors are also frequently cancer promoters [38]. The mechanisms used by inflammatory condition to promote tumor growth are numerous and extremely complex. Apart from enhanced proliferation or increased survival they may also involve the activation of angiogenesis and metastasizing. This is associated, among others, with the production by the transforming cancer cells of many cytokines and chemokines which attract various populations of immune cells, including macrophages, neutrophils, adipose cells, dendritic cells and T, B and NK cells, which in turn infiltrate tumor tissues and form tumor microenvironment [39]. Leukocytes may account for as many as $50 \%$ of total tumor mass. The interaction between cancer cells and macrophages stimulates them to produce such cytokines as IL-8, which stimulates further inflow of inflammatory cells. In tumor microenvironment, cells communicate with one another either by direct contact or production of various mediators [40].

Concluding, inflammatory processes can modulate the course of cancer, inhibiting or stimulating its growth. The activity of inflammatory cells, and the type and level of the inflammation-modulating factors affect the balance between their pro- and antitumor effects. In the initial phase of cancer growth, the acute phase responses may show anticancer action, as it should be remembered that the acute inflammatory reaction in itself is a physiological reaction developing in response to a damaging stimulus and aimed to eliminate the stimulus. It has been shown that in certain types of cancers the ability to induce controlled acute inflammatory condition is used as a therapy component. However, in a chronic inflammatory state, especially in tumor microenvironment, the presence of inflammatory cells acts rather for the benefit of cancer cells, stimulating their survival and proliferation.

The thorough knowledge and understanding of the mechanisms that associate inflammation with neoplastic disease may provide tangible benefits both in the scientific and clinical aspects related to the introduction of new diagnostic and therapeutic methods. The assessment of the involvement of inflammation in respective neoplastic lesions may affect the preventive and therapeutic measures in patients with chronic inflammations.

\section{Compliance with ethical standards}

Conflict of interest The authors have no conflicts of interest to disclose.

Ethical approval This article does not contain any studies with human participants or animals performed by any of the authors. 
Open Access This article is distributed under the terms of the Creative Commons Attribution 4.0 International License (http://crea tivecommons.org/licenses/by/4.0/), which permits unrestricted use, distribution, and reproduction in any medium, provided you give appropriate credit to the original author(s) and the source, provide a link to the Creative Commons license, and indicate if changes were made.

\section{References}

1. Virchov R (1863) Cellular pathology as based upon physiological and pathological histology. J. B. Lippincott, Philadelphia. doi:10. 5962/bhl.title.32770

2. Dvorak HF (1986) Tumors: wounds that do not heal. Similarities between tumor stroma generation and wound healing. N Engl J Med 315:1650-1659. doi:10.1056/NEJM198612253152606

3. Trinchieri G (2011) Inflammation in Cancer: a therapeutic target? Oncology (Williston Park) 25:418-420. doi:10.1016/S1470-2045 (11)70203-0

4. Kołaczkowska E (2007) Acute inflammation as a beneficial process - history and recent developments. Kosmos 56:27-38

5. Scott A, Khan KM, Cook JL et al (2004) What is "inflammation"? Are we ready to move beyond Celsus? Br J Sports Med 38:248-249. doi:10.1136/bjsm.2003.011221

6. Granger DN, Senchenkova E (2010) Historical perspectives. In: Inflammation and the microcirculation, Chapt. 2. Morgan \& Claypool Life Sciences, San Rafael (CA), pp 3-6. doi:10.4199/ C00013ED1V01Y201006ISP008

7. Ley K (2001) History of inflammation research. In: Physiology of inflammation. Methods in Physiology Series, Chapt. 1, Oxford University Press, pp 1-10. doi:10.1007/978-1-4614-7512-5

8. Grivennikov SI, Greten FR, Karin M (2010) Immunity, inflammation, and cancer. Cell 140:883-899. doi:10.1016/j.cell.2010. 01.025

9. Dmitrieva OS, Shilovskiy IP, Khaitov MR et al (2016) Interleukins 1 and 6 as main mediators of inflammation and cancer. Biochemistry 81:80-90. doi:10.1134/S0006297916020024

10. Zarbock A, Ley K, McEver RP et al (2011) Leukocyte ligands for endothelial selectins: specialized glycoconjugates that mediate rolling and signaling under flow. Blood 118:6743-6751. doi:10. 1182/blood-2011-07-343566

11. Wedepohl S, Beceren-Braun F, Riese S et al (2012) L-selectin-a dynamic regulator of leukocyte migration. Eur J Cell Biol 91:257-264. doi:10.1016/j.ejcb.2011.02.007

12. Mann AP, Tanaka T (2011) E-selectin: its role in cancer and potential as a biomarker. Transl Med Surg 1:002. doi:10.4172/ 2161-1025.S1-200

13. Muller WA (2013) Getting leukocytes to the site of inflammation. Vet Pathol 50:7-22. doi:10.1177/0300985812469883

14. Zarbock A, Kempf T, Wollert KC et al (2012) Leukocyte integrin activation and deactivation: novel mechanisms of balancing inflammation. J Mol Med 90:353-359. doi:10.1007/s00109-0110835-2

15. Kim M, Carman CV, Springer TA (2003) Bidirectional transmembrane signaling by cytoplasmic domain separation in integrins. Science 301:1720-1725. doi:10.1126/science.1084174

16. Mitroulis I, Alexaki VI, Kourtzelis I et al (2015) Leukocyte integrins: role in leukocyte recruitment and as therapeutic targets in inflammatory disease. Pharmacol Ther 147:123-135. doi:10. 1016/j.pharmthera.2014.11.008

17. Sumagin R, Sarelius IH (2010) Intercellular adhesion molecule-1 enrichment near tricellular endothelial junctions is preferentially associated with leukocyte transmigration and signals for reorganization of these junctions to accommodate leukocyte passage. J Immunol 184:5242-5252. doi:10.4049/jimmunol. 0903319

18. Schenkel AR, Mamdouh Z, Muller WA (2004) Locomotion of monocytes on endothelium is a critical step during extravasation. Nat Immunol 5:393-400. doi:10.1038/ni1051

19. Woodfin A, Voisin MB, Beyrau M et al (2011) The junctional adhesion molecule JAM-C regulates polarized transendothelial migration of neutrophils in vivo. Nat Immunol 12:761-769. doi:10.1038/ni2062

20. Muller WA (2007) PECAM: Regulating the start of diapedesis. In: Adhesion molecules: function and inhibition. Progress in inflammation research, pp 201-220. doi:10.1007/978-3-76437975-9-8

21. Ley K, Laudanna C, Cybulsky MI et al (2007) Getting to the site of inflammation: the leukocyte adhesion cascade updated. Nat Rev Immunol 7:678-689. doi:10.1038/nri2156

22. Bixel MG, Li H, Petri B et al (2010) CD99 and CD99L2 act at the same site as, but independently of, PECAM-1 during leukocyte diapedesis. Blood 116:1172-1184. doi:10.1182/blood-2009-12256388

23. Proebstl D, Voisin MB, Woodfin A et al (2012) Pericytes support neutrophil subendothelial cell crawling and breaching of venular walls in vivo. J Exp Med 209:1219-1234. doi:10.1084/jem. 20111622

24. Nagata M (2005) Inflammatory cells and oxygen radicals. Curr Drug Targets Inflamm 4:503-504. doi:10.2174/1568010054 526322

25. Kolaczkowska E, Kubes P (2013) Neutrophil recruitment and function in health and inflammation. Nat Rev Immunol 13:159-175. doi:10.1038/nri3399

26. Crusz SM, Balkwill FR (2015) Inflammation and cancer: advances and new agents. Nat Rev Clin Oncol 12:584-596. doi:10.1038/nrclinonc. 2015.105

27. Aggarwal BB, Sung B (2011) The relationship between inflammation and cancer is analogous to that between fuel and fire. Oncology (Williston Park) 25:414-418

28. Askeland EJ, Newton MR, O'Donnell MA et al (2012) Bladder cancer immunotherapy: BCG and beyond. Adv Urol 2012:181987. doi:10.1155/2012/181987

29. Salem ML, Attia ZI, Galal SM (2016) Acute inflammation induces immunomodulatory effects on myeloid cells associated with anti-tumor responses in a tumor mouse model. J Adv Res 7:243-253. doi:10.1016/j.jare2015.06.001

30. Balkwill FR, Mantovani A (2012) Cancer-related inflammation: common themes and therapeutic opportunities. Semin Cancer Biol 22:33-40. doi:10.1016/j.semcancer.2011.12.005

31. Eiró N, Vizoso FJ (2012) Inflammation and cancer. World J Gastrointest Surg 4:62-72. doi:10.4240/wjgs.v4.i3.62

32. Aggarwal BB, Shishodia S, Sandur SK et al (2006) Inflammation and cancer: how hot is the link? Biochem Pharmacol 72:1605-1621. doi:10.1016/j.bcp.2006.06.029

33. Coussens LM, Werb Z (2002) Inflammation and cancer. Nature 420:860-867. doi:10.1038/nature01322

34. Mantovani A, Allavena P, Sica A et al (2008) Cancer-related inflammation. Nature 454:436-444. doi:10.1038/nature07205

35. Meira LB, Bugni JM, Green SL et al (2008) DNA damage induced by chronic inflammation contributes to colon carcinogenesis in mice. J Clin Invest 118:2516-2525. doi:10.1172/ JCI35073

36. Okazaki IM, Kotani A, Honjo T (2007) Role of AID in tumorigenesis. Adv Immunol 94:245-273. doi:10.1016/S0065-2776(06) 94008-5

37. Sakurai T, He G, Matsuzawa A et al (2008) Hepatocyte necrosis induced by oxidative stress and IL-1 alpha release mediate 
carcinogen-induced compensatory proliferation and liver tumorigenesis. Cancer Cell 14:156-165. doi:10.1016/j.ccr.2008.06.016

38. Karin M (2006) Nuclear factor-kappaB in cancer development and progression. Nature 441:431-436. doi:10.1038/nature04870

39. de Visser KE, Eichten A, Coussens LM (2006) Paradoxical roles of the immune system during cancer development. Nat Rev Cancer 6:24-37. doi:10.1038/nrc1782
40. Liang W, Ferrara N (2016) The complex role of neutrophils in tumor angiogenesis and metastasis. Cancer Immunol Res 4:83. doi:10.1158/2326-6066.CIR-15-0313 